\title{
Strength Prediction of Adhesively Bonded Single Lap Joints with the eXtended Finite Element Method
}

\author{
O. Völkerink*, J. Kosmann, M. J. Schollerer, D. Holzhüter, C. Hühne \\ German Aerospace Center (DLR), Institute for Composite Structures and Adaptive \\ Systems, Braunschweig, Germany
}

\begin{abstract}
Because of a planar load introduction, a high strength over weight ratio, avoidance of holes, corrosion resistance and component reduction, adhesively bonded joints attract increasing attention in lightweight construction. Nonetheless, a reliable prediction of the joints' behaviour in terms of stress and strength is still a tremendous challenge.

In this study the eXtended Finite Element Method (XFEM) in combination with cohesive behaviour is used to model crack growth under quasi-static loading. The objective of this work is to study the influence of different continuum mechanical material models for the adhesive on XFEM strength predictions. It will be evaluated whether the strength prediction of joints mainly loaded in shear is more accurate with a high sophisticated material model. Therefore, strength predictions of Single Lap Joints (SLJ) with thick aluminium adherends are performed. Herein, digital image correlation (DIC) data recorded during experimental tests are used to assess the material models in combination with the derived material parameters. Furthermore, with the experimental results the performed strength predictions are evaluated. It could be revealed that XFEM in combination with a suitable yield criterion and appropriate material parameters is a valid technique for the strength prediction of lap shear joints since the variance to the experiments is less than $1 \%$.
\end{abstract}

\footnotetext{
* Corresponding author

Email address: oliver.voelkerink@dlr.de (O. Völkerink)
}

Preprint submitted to The Journal of Adhesion

November 25, 2018 
Keywords: XFEM, Drucker-Prager, lap-shear, finite element analysis, aerospace, damage mechanics

\section{Introduction}

Bonded joints in present structural applications do not utilise their full load carrying potential. Methods, used to size adhesively bonded joints are associated with the use of high safety factors because their strength predictions suffer

5 from a great uncertainty. On that account, this research explores the eXtended Finite Element Method (XFEM) for the strength prediction of mainly shear loaded bonded joints.

The significance of bonded joints for connecting structural components increases particularly within the field of lightweight construction. The automobile manufacturer BMW utilises these benefits for the assembly of components made out of fibre reinforced plastics. The total length, of all bondlines within the car body of the BMW i8, sums up to approximately $150 \mathrm{~m}$ [1]. Bonded joints are further used on a large scale within rotor blades of wind turbines [2, between spars and aerodynamic skin. An equal example that shows the necessity of 15 adhesive joint design within a global structural consideration is the unmanned aircraft system SAGITTA, where all connections are realised as adhesive joints [3. In the design phase of such bonded structures a reliable strength prediction of different joint configurations is mandatory.

To predict the strength of bonded joints one can apply analytical or numerical methods. An overview of available linear and nonlinear analytical approaches is given by da Silva et al. [4, 5] and Rodriguez et al. [6. Linear analytical analyses give safe predictions but are usually very conservative. Whereas nonlinear analyses are very expensive and the advantage over Finite Element Analysis (FEA) is disputable. In addition, the analytical models are only suitable for the analysis of single joints. Unlike the state-of-the-art analytical models, continuum mechanical finite element based approaches are appropriate for global structural design. Reviews of design methods for adhesive joints that base are 
based on FEA are given by He [7] and Jia [8. Continuum mechanics modelling relies on the comparison of the actual stress or strain state to a limit value [9]. Therefore, damage growth cannot be modelled.

Tomblin et al. [10 state that the majority of industrial practitioners verify the adequacy of the adhesive joint design by analysing average shear stresses. Several requirements have to be met to ensure that this approach is valid. The overlap length cannot be increased arbitrarily to reduce the average shear stress, to a certain amount with this measure. Thus, with this method also high safety factors have to be used. The application of the less complex analytical and continuum mechanical methods, however, is associated with the use of reduction factors, which prevent from utilising the full load carrying potentials of the 40 adhesive joint.

In order to overcome these limitations, researchers use damage mechanics approaches to be able to model damage initiation and propagation due to local stress concentrations. Extended reviews about available methods are presented by Pascoe et al. [11] and de Sousa et al. [12. The Cohesive Zone Modeling 45 (CZM) 13] and the Virtual Crack Closure Technique (VCCT) 14 are common methods to incorporate damage mechanics in FEM models. While VCCT is eligible for very brittle materials only, CZM is the common approach for adhesive joints. Another promising approach for failure prediction of adhesive joints is the use of Smoothed Particle Hydrodynamics (SPH) to model cohesive cracks in the adhesive. However, Mubashar and Ashcroft [15] state that SPH requires further development to compete with existing methods like CZM in terms of stress prediction.

Whereas the crack path in analysis with CZM is defined a priori because special purpose elements are needed, in combination with XFEM, cracks can grow arbitrarily in the finite element model without the need of remeshing. A detailed description of XFEM is given in section 3.4. Campilho et al. [16] performed strength predictions of single and double lap joints with a brittle adhesive and aluminium adherends with overlap length $l$ between 5 and $20 \mathrm{~mm}$. With the 
maximum principal stress criterion used for crack initiation, damage growth could not be simulated with XFEM. Mubashar et al. [17] used XFEM to model cracks in the adhesive fillet region of single lap joints and CZM for the interfacial region. Stuparu et al. [18] applied the same combination of XFEM for modelling cracks in the adhesive and CZM for the interface. Both studies showed that crack growth in the fillet could be well modelled. Although like Campilho et al. 16 they also showed that XFEM with a maximum principal stress criterion for crack initiation is not able to model crack propagation in the adhesive layer as the crack growth towards the adherends. This behaviour does not represent the experimental observations. Xará and Campilho [19] studied the influence of different XFEM damage initiation criteria on the strength prediction. They 70 showed that the maximum principal stress criterion used by Campilho et al. [16] and Mubashar et al. [17] is the most inappropriate one. With the quadratic stress criterion the difference in strength between experiment and numerical prediction is less than $10 \%$. Apart from this, they also performed strength predictions with CZM and observed that the computation times between CZM 75 and XFEM are comparable. All of the studies mentioned, used von Mises as elastic-plastic material model for the adhesives.

The objective of this work is to study the influence of different continuum mechanical material models for the adhesive like the von Mises (vM) [20], linear (IDP) and exponent Drucker-Prager (eDP) model [21] on the XFEM strength

${ }_{80}$ prediction. It will be evaluated whether the strength prediction of joints mainly loaded in shear is more accurate with a high sophisticated material model. To achieve this, at first comparisons are made between the strain distributions in the adhesive bondline of Thick Adherend Shear Tests (TAST) specimen derived from numerical simulations and experimental data recorded with the digital 85 image correlation technique. Then, strength predictions using $2 \mathrm{D}$ plain strain XFEM simulations with different material models are conducted. In this vein, the influence of the mesh density on the strength prediction is investigated. 


\section{Experimental Work}

\subsection{Adherends and Adhesives}

In the experimental work TAST specimen are tested to evaluate the failure behaviour of a film adhesive under shear loading. The adherends are made out of aluminium sheets (EN AW 5083). In the numerical simulations the aluminium parts are assumed to be linear elastic with a young's modulus $E$ of $71000 \mathrm{MPa}$ and a poisson ratio $\nu$ of 0.3322 . The specimen are bonded with the LOCTITE EA 9695050 NW AERO epoxy film adhesive (Henkel Corporation, Rocky Hill, CT, USA). The properties required for the numerical simulations are summarised in table 1 and were obtained both from own experiments and from literature. Young's Modulus $E$ and poisson ratio $\nu$ are determined from own dogbone specimen tests [23]. Likewise, the data for the shear hardening curve are obtained from own tubular butt joint specimen tested at Fraunhofer IFAM under pure torsion loading conditions 24]. The yield stresses under various load combinations which are necessary to fit the different yield criteria, are taken from butt joint (BJ), inclined butt joint (IBJ) and thick adherend shear joint (TASJ) test results published by Nagel and Klapp [25]. The values necessary to model the cohesive behaviour of the XFEM cracks such as the shear strength $t_{s}^{0}$ and the strength in tension $t_{t}^{0}$ are also taken from Nagel and Klapp [25, while the thickness-dependant fracture toughnesses in tension and shear $\left(G_{I C}\right.$ and $\left.G_{I I C}\right)$ are taken from Floros et al. 26. The latter used double cantilever beam and end-notch flexure specimens with a bondline thickness of $t_{b}=0.15 \mathrm{~mm}$.

This value lies in between the two bondline thicknesses considered in this work $\left(t_{b}=0.1 \mathrm{~mm}\right.$ and $\left.t_{b}=0.2 \mathrm{~mm}\right)$. Therefore, the fracture toughnesses from [26] can be used as an approximation.

\subsection{Manufacturing of the Specimen}

The geometry of the TAST specimen is represented in figure 1 and was chosen according to ASTM D 5656 [27]. First, the aluminium plates with a thickness of $10 \mathrm{~mm}$ were sandblasted with white corundum (grain number F180) and 
Table 1: Properties of LOCTITE EA 9695050 NW AERO epoxy film adhesive 23, 24, 25, 26]

\begin{tabular}{ll}
\hline Property & \\
\hline Young's modulus, $E[\mathrm{MPa}]$ & 2205.6 \\
Poisson's ratio, $\nu[-]$ & 0.361 \\
Tensile yield strength, $\sigma_{y}[\mathrm{MPa}]$ & 46.78 \\
Tensile failure strength, $\sigma_{f}[\mathrm{MPa}]$ & 51.28 \\
Shear yield strength, $\tau_{y}[\mathrm{MPa}]$ & 32.78 \\
Shear failure strength, $\tau_{f}[\mathrm{MPa}]$ & 51.94 \\
Fracture toughness in tension, $G_{I C}[\mathrm{~N} / \mathrm{mm}]$ & 1.019 \\
Fracture toughness in shear, $G_{I I C}[\mathrm{~N} / \mathrm{mm}]$ & 0.783 \\
\hline
\end{tabular}

then cleaned with acetone and isopropyl alcohol. After the surface preparation, the adhesive film was applied and the bonded aluminium plates were vacuum bagged for one hour. For an adhesive bondline thickness of $t_{b}=0.1 \mathrm{~mm}$ one layer of adhesive film and for a thickness of $t_{b}=0.2 \mathrm{~mm}$ two layers were used. The curing of the adhesive was performed in a heat press with temperature and pressure according to the data sheet of the adhesive [28] in an evacuated press chamber. After curing, the plates were cut in strips with a band saw and the final specimen geometry including the two holes were machined with a CNC mill.

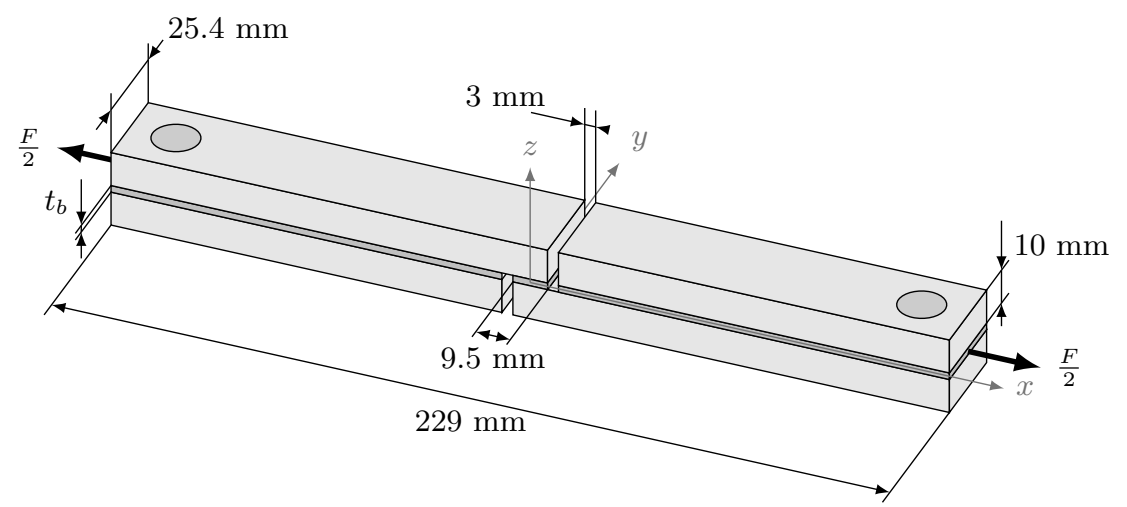

Figure 1: Sketch of Specimen Geometry 


\subsection{Experimental Tests}

The tensile testing of the TAST specimen was performed at room temperature in a Zwick 1476 servo-mechanic testing machine equipped with a 100 $\mathrm{kN}$ load cell . The tests were carried out with a constant crosshead speed of $v_{c h}=0.05 \mathrm{in} / \mathrm{min}$. For each of the two configurations four specimen were tested. In addition to the load-displacement data gathered, data for digital image correlation (DIC) were recorded with a consumer full-frame mirrorless camera and a macro lens to get full-field strain fields of the adhesive layer. The camera was coupled to the testing machine with a self developed interface box in order to correlate the data of the load cell with the recorded images. Afterwards, the recorded image data were evaluated with the Software Correlate Professional 2017 from GOM. A detailed description of the Digital Image Correlation (DIC) setup and the data processing can be found in Kosmann et al. 29].

\section{Numerical Work}

\subsection{Conditions of the Analyses}

All presented analyses were carried out with Abaqus/Standard as implicit dynamic analysis using direct integration in order to improve convergence. For this type of analysis the solver assigns numerical settings based on an application given by the user. This application type was classified as quasi-static for all simulations. The study regarding the influence of the material models, the discretisation and the bondline thickness on the accuracy of the XFEM strength prediction was performed with $2 \mathrm{D}$ FE-models. The adhesive layer as well as the adherends were modelled with reduced integrated linear plain strain elements (CPE4R). The interface between adhesive and adherends was modelled via shared nodes. The mesh density of the adherends changes with a single bias in $\mathrm{x}$ - and $\mathrm{y}$-direction from the overlap area towards the ends of the specimen to $1 \mathrm{~mm}$ by $1 \mathrm{~mm}$. Unless otherwise noted, all simulations were performed without viscous regularisation. Figure 2 shows the geometry of the model and 
the boundary conditions. In order to model the exact adhesive bondline thicknesses of the experimental tested specimen in the finite element analyses the thicknesses were measured in microsections of the TAST specimen. As a result thicknesses of $t_{b}=0.125 \mathrm{~mm}$ and $t_{b}=0.21 \mathrm{~mm}$ were used. The simulations were performed with a displacement load $\delta$ and terminated when a significant load drop induced from the XFEM crack in the adhesive occurred. The loads were calculated from the sum of the reaction forces at the nodes from the rigidly clamped specimen end. The maximum of the sum of the reaction forces was then taken as the numerical failure load.

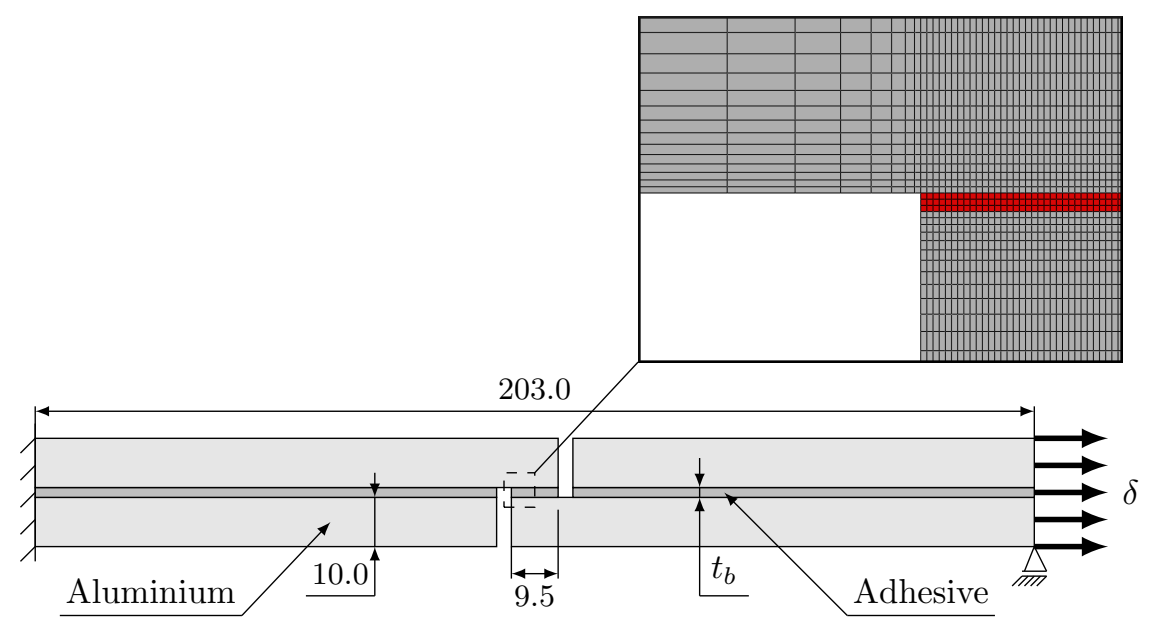

Figure 2: Geomentry, dimensions and boundary conditions of 2D FE-model

Although 2D plain strain simulations are less time consuming, 3D analyses were also performed. The reason for the two different kinds of simulations is that the strains conducted by the DIC system were measured at the side surface of the specimen. The measured strains in this area cannot be compared to strains from a $2 \mathrm{D}$ plain strain analysis which represents a mid-section cut through the specimen without any further verification. For this reason also 3D simulations were performed. In order to determine the discretisation of the adhesive layer a mesh convergence study was performed. The study was conducted with one, three, five and seven linear brick elements (C3D8) in through-thickness direction 
and an aspect ratio between 1.0 and 1.4. The analysis with one element shows the smallest peak stresses at the overlap edges, whereas the analysis with three elements yields the largest peak stresses. Results from simulations with five and seven elements show a very similar stress distribution. As compromise between accuracy and computational costs five elements in through-thickness direction was chosen for discretisation. The adherends are modelled by reduced integrated C3D8R linear brick elements with a edge length of $1.0 \mathrm{~mm}$. As can be seen in figure 3 to save computation time, the model takes advantages of the symmetry in the x-z-plane of the specimen. The interface between adhesive and adherend was modelled with a tied contact.

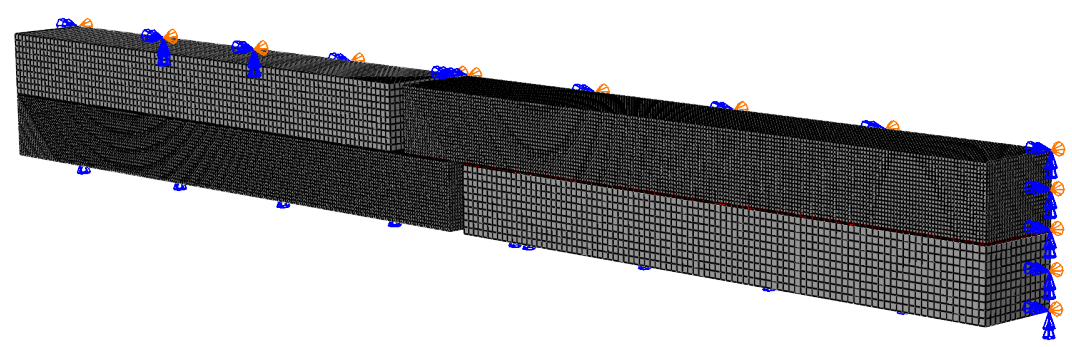

Figure 3: 3D FE-model, half model using x-z symmetry plane, tied contact between adhesive and adherends

\subsection{Elastic-Plastic Material Models}

Prior to damage in the form of discrete cracks, adhesives show inherent nonlinear material behaviour. Therefore, elastic-plastic material models are used to model the adhesives behaviour until fracture in finite element analyses. In order to predict the transition between the elastic and the plastic regime, all of these material models require a suitable yield criterion which is valid for the adhesive under consideration [30]. In contrast to metals, the plastic behaviour of adhesives is sensitive to the hydrostatic stress component [9]. For this reason, a suitable yield criterion has to take into account the influence of the hydrostatic stress on the yield point. Without any claim to completeness, three possible yield criteria for the simulations in this work will be discussed in the following. 


\subsection{1. von Mises}

The von Mises yield criterion [20] states that yielding starts when the equivalent stress $q$ or rather the second deviatoric invariant $J_{2}$ reaches the critical values $\sigma_{y}$ [20], cf. equation 3.1 .

$$
F_{v M}=\sqrt{3 J_{2}}-\sigma_{y}=q-\sigma_{y}=0
$$

Since the von Mises material model does not account for the influence of the hydrostatic component of stress on the yield point, it does not fullfill the demand stated in section 3.2 and is not well suited for adhesives. Nonetheless, it will be the baseline for the simulations in this work because of its easy parameter setting. As shown in figure 4. only pure shear tests are necessary to estimate the model parameters. For the simulations in this work $\sigma_{y}$ is set to $51.85 \mathrm{MPa}$.

\subsubsection{Linear Drucker-Prager}

A yield criterion where the onset of yielding is linearly dependant from the hydrostatic stress $p$ is the linear Drucker-Prager criterion [21, cf. equation 3.2 .

$$
F_{\text {lin } D P}=q-p \cdot \tan (\beta)-d=0
$$

The sensitivity of yielding to the hydrostatic stress $p$ is characterised by the hydrostatic stress sensitivity parameter $\beta$ being material dependant. The parameter $d$ is related to the shear yield stress $\tau_{y}$ with $d=\frac{\sqrt{3}}{2} \tau_{y}\left(1+\frac{1}{K}\right)$.

According to the results of Dean et al. 30] the linear Drucker-Prager model is improper for the investigated epoxy adhesives but appears to describe the behaviour of the investigated acrylic adhesives by Dean et al. [30. Although the adhesive under consideration in this work is epoxy-based, the linear DruckerPrager criterion will be considered, since Dean et al. 30] stated that the findings could be fortuitous. The chosen material parameters are presented in table 2 
Table 2: Linear Drucker-Prager parameters for LOCTITE EA 9695050 NW AERO epoxy film adhesive

\begin{tabular}{ll}
\hline Property \\
\hline Hydrostatic stress sensitivity parameter, $\beta\left[{ }^{\circ}\right]$ & 39.08 \\
Cohesion parameter, $d[\mathrm{MPa}]$ & 51.85 \\
\hline
\end{tabular}

210

\subsubsection{Exponent Drucker-Prager}

In figure 4 the yield stresses of the three different test configurations are expressed in terms of equivalent stress $q$ and hydrostatic stress $p$. In addition, the yield surfaces described by the von Mises and the linear Drucker-Prager Model with the chosen material parameters are incorporated. Although the linear Drucker-Prager model takes into account the hydrostatic stress $p$ for the onset of yielding, it can be seen that the criterion cannot describe all three test configurations since the test data do not lie on a straight surface. For this reason, the exponent Drucker-Prager criterion [21] is considered as a third yield criterion in this work. The exponential Drucker-Prager criterion forms a hyperbolic yield surface with the material parameter $a$, the exponent parameter $b$ and the hardening parameter $p_{t}$. The yield surface can be described with the following equation:

$$
F_{e x p D P}=a q^{b}-p-p_{t}=0
$$

In order to determine the parameters $a, b$ and $p_{t}$ for the exponential DruckerPrager yield criterion the flow function 3.3 is fitted through the mean yield stresses from the TASJ, BJ and IBJ tests 25] with a least square fit using a Python script. The fitted function is shown in figure 4 and the estimated values for the parameters $a, b$ and $p_{t}$ are summarised in table 3

\subsection{Hardening Curve}

To describe the material behaviour after onset of yielding, a hardening curve must be given to Abaqus as an input. In this work, the hardening curve was gained from tubular butt joint specimen built at DLR-Institute of Composite 
Table 3: Fitted exponential Drucker-Prager parameters for LOCTITE EA 9695050 NW AERO epoxy film adhesive

\begin{tabular}{ll}
\hline Property & \\
\hline Material parameter, $a[-]$ & 0.00219 \\
Exponent parameter, $b[-]$ & 2.448 \\
Hardening parameter, $p t[\mathrm{MPa}]$ & 43.082 \\
\hline
\end{tabular}

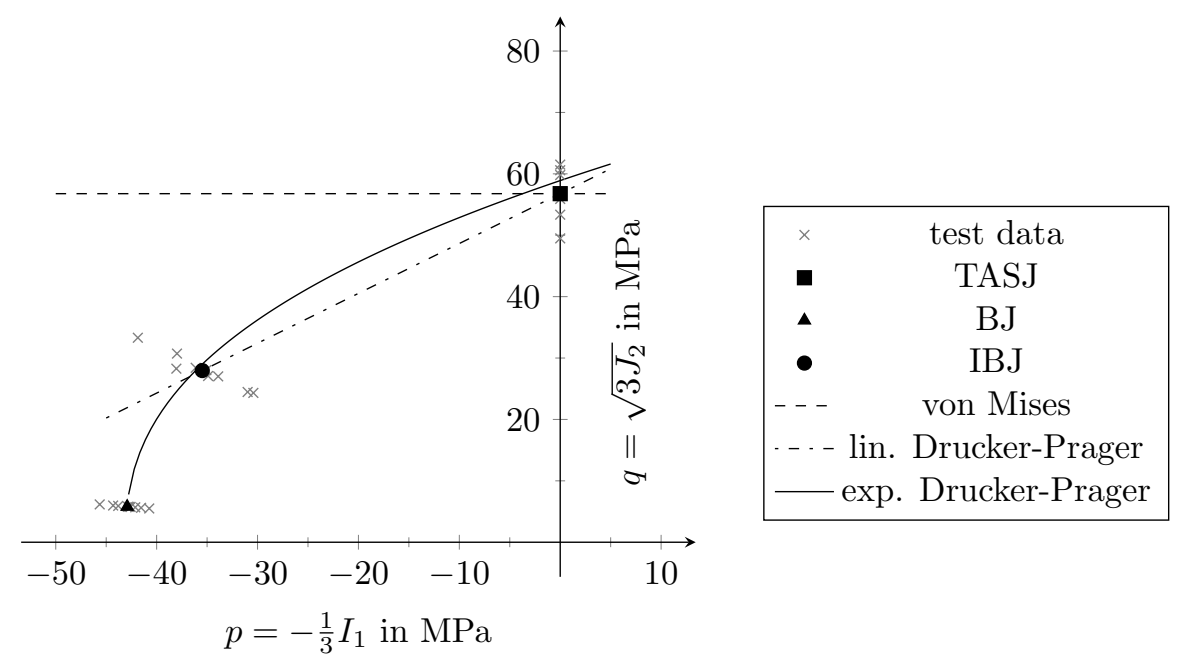

Figure 4: Yield data expressed in hydrostatic $p$ and deviatoric $q$ stresses

Structures and Adaptive Systems' Composite Joining Lab [31] and tested under torsion loading at Fraunhofer IFAM. The $\tau$ - $\gamma$-data derived from the test are shown in figure 5 (a).

At first, the yield stress $\tau_{y}$ has to be determined to obtain a shear stress - plastic strain curve. These data were then used to calculate a true stress - true strain curve according to Dean and Crocker [32. The hardening curve for the DruckerPrager models has to be given in terms of cohesion $d$ and effective true plastic strain $\varepsilon_{e f f}^{p}$. In contrast, the hardening curve for the von Mises model has to be specified in Abaqus in terms of effective stress $\sigma_{e f f}$ and effective plastic strain $\varepsilon_{e f f}^{p}$. The data can be converted with the equations $3.4-3.6$.

$$
\sigma_{e f f}=\sqrt{3} \tau
$$




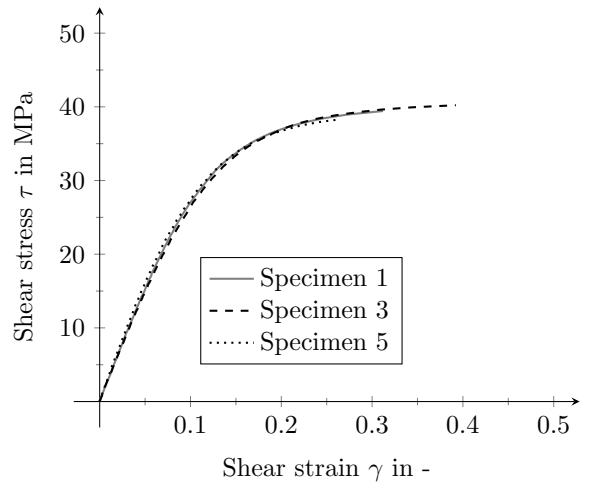

(a) Experimental data

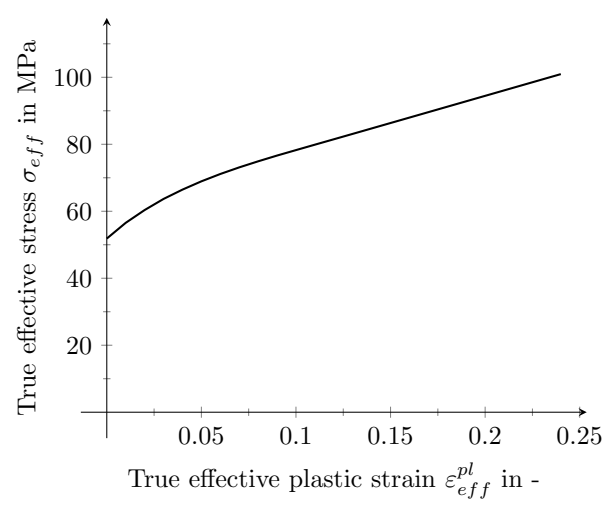

(b) Simulation input

Figure 5: Hardening curve

$$
\begin{aligned}
& d=\frac{\sqrt{3}}{2} \tau\left(1+\frac{1}{K}\right) \\
& \varepsilon_{\text {eff }}^{p}=\frac{1}{\sqrt{3}} \gamma^{p}
\end{aligned}
$$

The yield surface shape in the deviatoric stress plane is described by the parameter $K$. In this work, the parameter $K$ is set to 1 which means that the flow stress in triaxial tension is equal to the flow stress in triaxial compression [33]. In this case, the hardening curve for the von Mises and the Drucker-Prager models are the same. The final hardening data used for all simulations are shown in figure 5 (b).

\section{4. eXtended Finite Element Method}

The XFEM is an extension of the classic FEM, which allows to model discrete cracks in a continuum through an enrichment of the displacement field with discontinuous functions, cf. 3.7, developed by Belytschko and Black 34. These enrichment functions allow the inclusion of a priori not known fracture planes 
and singular expressions in the existing finite element mesh.

$$
\boldsymbol{u}^{\boldsymbol{h}}=\sum_{i=1}^{N} N_{i}(x) \boldsymbol{u}_{\boldsymbol{i}}+\sum_{i=1}^{N} M_{i}(x) a_{i}
$$

The approximation of the XFEM displacement field $\boldsymbol{u}^{\boldsymbol{h}}$ consists of the standard FEM function at node i $N_{i}(x)$ and the related unknown $\boldsymbol{u}_{\boldsymbol{i}}$ as well as the enrichment part with the local enrichment function $M_{i}(x)$ and the unknown of the enrichment $a_{i}$ both at node i 35 . The enrichment is only active when a crack exists and enables the establishment of phantom nodes which subdivide the element into two subelements formed by the original and the phantom nodes. The displacement fields of these two subelements are completely independent from each other and replace the original element. Whether or not a crack exists in a finite element depends on the evaluation of a crack initiation criteria. In Abaqus 2016 [36] six different stress or strain based criteria are available. For this work, only the quadratic nominal stress criterion (QUADS), cf. 3.8 is considered as it is stated to be best suited for the most joint/adhesive configurations by Xará and Campilho [19].

$$
f=\left\{\frac{\left\langle t_{n}\right\rangle}{t_{n}^{0}}\right\}^{2}+\left\{\frac{t_{s}}{t_{s}^{0}}\right\}^{2}+\left\{\frac{t_{t}}{t_{t}^{0}}\right\}^{2}
$$

The formed subelements are allowed to separate from each other according to a suitable cohesive law. In the simulations presented cohesive zones with an energetic failure power law criterion, cf. 3.9, are used to model the crack progression [36]. All analyses were carried out with an exponent $\alpha=1$ which leads to a linear softening law.

$$
\left(\frac{G_{I}}{G_{I C}}\right)^{\alpha}+\left(\frac{G_{I I}}{G_{I I C}}\right)^{\alpha}+\left(\frac{G_{I I I}}{G_{I I I C}}\right)^{\alpha}=1
$$

The material parameters used in this work for the XFEM modelling are summarised in table 4 
Table 4: Parameters of LOCTITE EA 9695050 NW AERO for XFEM modelling [19, 25, 26]

\begin{tabular}{ll}
\hline Property & \\
\hline$G_{I C}[\mathrm{~N} / \mathrm{mm}]$ & 1.019 \\
$G_{I I C}[\mathrm{~N} / \mathrm{mm}]$ & 0.783 \\
$G_{I I I C}[\mathrm{~N} / \mathrm{mm}]$ & 0.783 \\
$t_{n}^{0}[\mathrm{MPa}]$ & 51.28 \\
$t_{s}^{0}[\mathrm{MPa}]$ & 51.94 \\
$t_{t}^{0}[\mathrm{MPa}]$ & 51.94 \\
Damage Initiation Criterion & QUADS \\
Damage Propagation Criterion & Energetic Power Law \\
$\alpha$ & 1 \\
\hline
\end{tabular}

\section{Results and Discussion}

\subsection{Experimental Force-Displacement-Curve and Failure Assessment}

In figure 6 the typical fracture surfaces of the tested TAST specimen are shown for the bondline thickness of $t_{b}=0.21 \mathrm{~mm}$ as example. It can be observed that there is adhesive on both adherend surfaces which means that a cohesive failure in the bondline occured. It may be concluded that the surface preparation before bonding and the curing of the adhesive was sufficient.

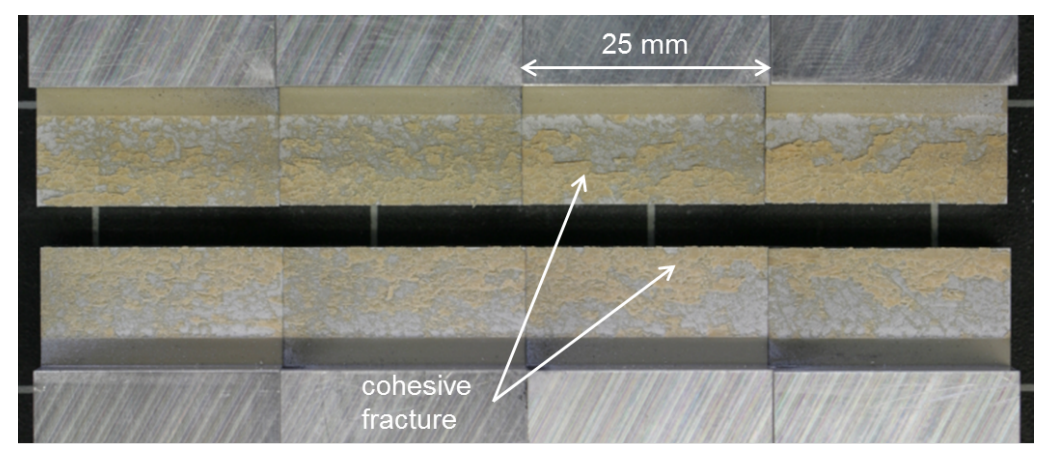

Figure 6: Failure surface of the specimen with $t_{b}=0.21 \mathrm{~mm}$

To add, each adherend has one overlap edge with more adhesive left on the surface than the other side and a transition zone in the middle. Therefore, it can be deduced from the fracture pattern that the cracks started near the interface at both overlap edges and then changed the direction in the middle of 
the overlap length. This behaviour is schematically shown in figure 7 and can be seen mainly in brittle adhesives 37 .

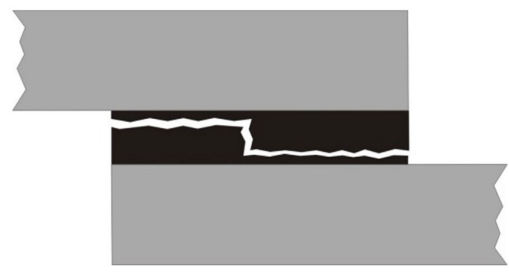

Figure 7: Crack propragation in the adhesive layer, from 38.

In figure 8 the recorded crosshead displacement-force data are plotted. It can be seen that all specimen lie very close together and also that there is not much difference between the two different bondline thicknesses. The determined failure loads are $F_{f}=11993 \pm 193 \mathrm{~N}$ for the thin bondline and $F_{f}=11607 \pm 463$ $\mathrm{N}$ for the thicker bondline. These findings are in agreement with the general relation that thinner bondlines bear higher loads when loaded in shear 39].

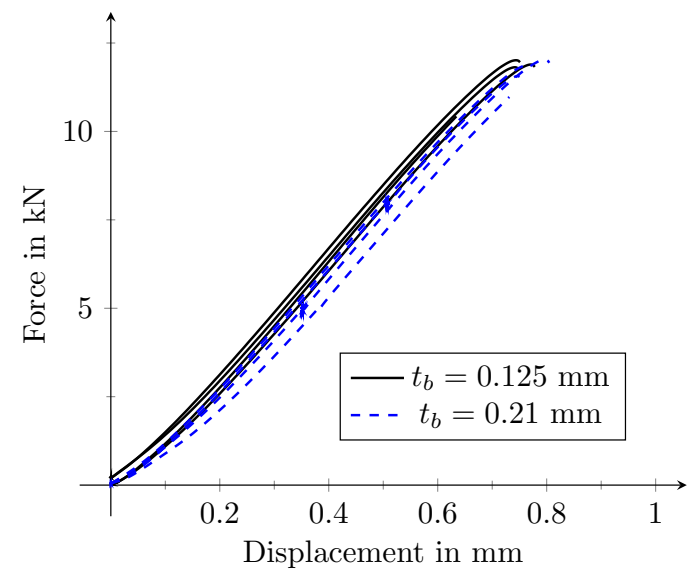

Figure 8: Force-displacement data from experimental tests

\subsection{Strain Distribution in the Adhesive Layer}

As mentioned in section 2.3 during the experimental tests DIC data were recorded. The resolution of the chosen setup enables the computation of several strain data points in thickness direction of the adhesive layer during post 


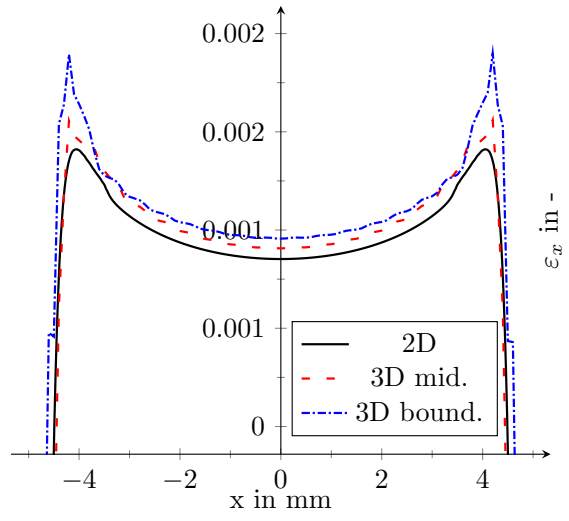

(a) $\varepsilon_{x}$

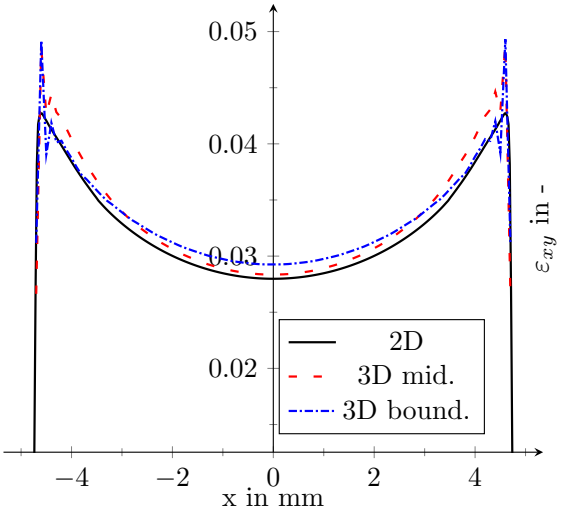

(b) $\varepsilon_{x y}$

Figure 9: Strain comparison of the FE models at $6.0 \mathrm{kN}$ with exponent Drucker-Prager model

processing of the DIC data. These data were used to compare the strain distribution of the FE-model calculated with the three different material models presented in 3.2 against the experimental data. In doing so, the material models in combination with the chosen material parameters can be validated.

In figure 9 a comparison between the $\varepsilon_{x^{-}}$and $\varepsilon_{x y}$-distribution along the overlap length in the adhesive layer between the $2 \mathrm{D}$ and the $3 \mathrm{D}$ FE-model at a loading of $F=6 \mathrm{kN}$ is shown. Both models were calculated with the exponent DruckerPrager model. The strains were taken from the element row in the middle of through thickness direction of the bondline. For the 3D model two strain distribution are plotted. One strain distribution was taken in the middle and one at the side surface of the specimen. The results show that there is little difference in shear strain between the two positions in the 3D model. For the reason that the DIC data were also captured at the side surface of the specimen, this element row will be taken into account for the comparison. Furthermore, the strain comparison in figure 9 shows that the simplified 2D plain strain model is also able to model the behaviour of the TAST specimen and can be used for the XFEM strength prediction in the following section.

In figures 10 (a) and (b) the strains $\varepsilon_{x}$ and $\varepsilon_{x y}$ are plotted at a loading of $1.5 \mathrm{kN}$. At this loading the material behaviour is assumed to be linear-elastic 


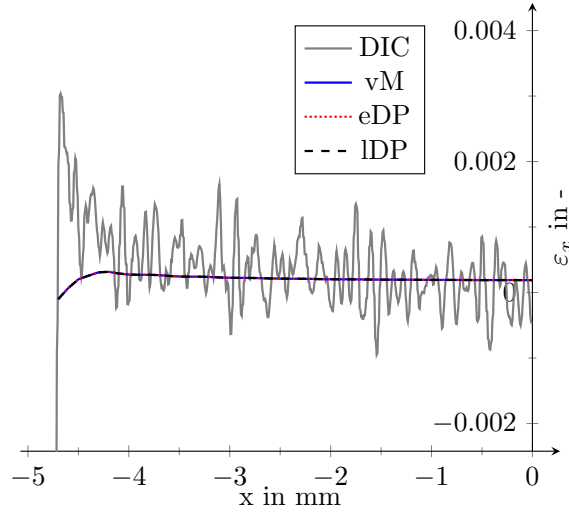

(a) $\varepsilon_{x}$

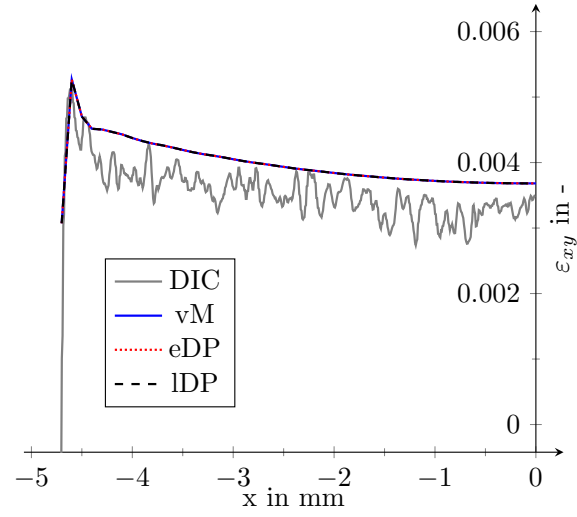

(b) $\varepsilon_{x y}$

Figure 10: Strain comparison at $1.5 \mathrm{kN}$

because the calculated stresses are lower than the yield stresses presented by Nagel and Klapp [25]. The simulations for this comparison were performed without XFEM crack modelling. Because the strains in y-direction $\varepsilon_{y}$ are about zero, they are not presented. The strains are plotted from the edge of the bondline at $x=-4.75 \mathrm{~mm}$ until the half overlap length $x=0 \mathrm{~mm}$ because the measurement field of the DIC system covers only $7.2 \mathrm{~mm}$ by $4.8 \mathrm{~mm}$. It is apparent that there is some noise in the DIC strain data. In this context, it is necessary to mention that the calculated strain data were not filtered in post-processing. Possible reasons for the noise are a high signal-to-noise ratio of the consumer camera's color sensor and / or diffraction of the lens caused by a small aperture. Nonetheless, the strains $\varepsilon_{x}$ are in good agreement with the experimental data. In comparison with the experiment, the shear strains $\varepsilon_{x y}$ are slightly overestimated by all three simulations. In addition, it can be noted that all three material models give the same strains. This is an expected result as the three materials models do not differ from each other in the linear-elastic regime.

In order to compare strain fields after the onset of yielding in the figures 11 (a) and (b) the strains $\varepsilon_{x}$ and $\varepsilon_{x y}$ are plotted at a loading of $F=6 \mathrm{kN}$. As with the lower loading the DIC strain data scatter, but the scatter especially of 


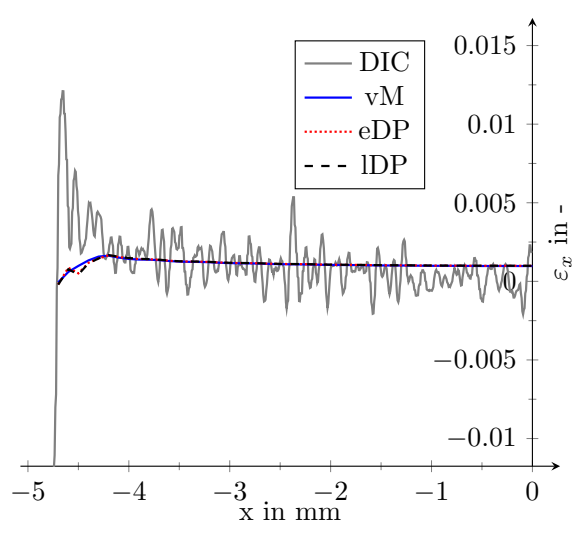

(a) $\varepsilon_{x}$

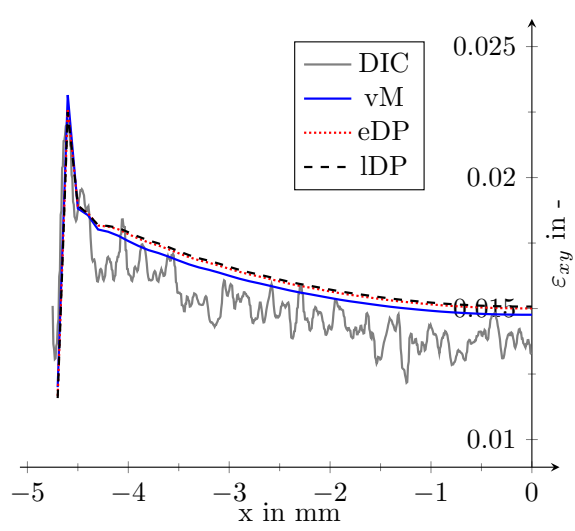

(b) $\varepsilon_{x y}$

Figure 11: Strain comparison at $6.0 \mathrm{kN}$

$\varepsilon_{x}$ is lower compared to figure 10. This is an expected result since the strain values are higher and therefore the noise has less influence. Like the findings for the $1.5 \mathrm{kN}$ loading the $\varepsilon_{x}$ strains from the experiment and the numerical simulations are in good agreement. Also, the numerical data only differ slightly at the outer boundary of the overlap at $x=-4.75 \mathrm{~mm}$. The $\varepsilon_{x y}$-values of all three simulations are again marginally higher than the experimental values. The von Mises model corresponds most closely to the experimental measured strains. As yielding had taken place at $F=6 \mathrm{kN}$ the difference between the numerical strain predictions can be explained with the different yield criteria.

As a result, it can be concluded that all three material models can describe the experimental behaviour adequately. The slight difference in the $\varepsilon_{x y^{-}}$ distribution might occur from measurement inaccuracy or the material parameters since the DIC measurements were performed on specimens with a bondline thickness of $t_{b}=0.2 \mathrm{~mm}$ and the parameter identification was performed with specimens with $t_{b}=0.1 \mathrm{~mm}$. Further testing with a more extensive amount of specimen has to be conducted. The good performance of the von Mises model could be traced back to the low hydrostatic stress component in the TAST specimen [40]. 


\subsection{XFEM Strength Prediction}

In order to save computation time the strength prediction study has been carried out with the 2D plain strain model described in section 3.1 as the stress and strain distribution is in good agreement with the 3D model, cf. section 4.2 . The through-thickness discretisation of the bondline was varied between 1 to 7 elements. The element length in $\mathrm{x}$-direction was selected in a way that the elements had a nearly quadratic shape.

Figure 12 shows a typical crack pattern from the XFEM analyses. The cracks grow near the interface from the opposite diagonal overlap edge towards the center of the bondline. On that note, the XFEM crack pattern is in good agreement with the experimental findings. In figure 13 the resulting load-displacement data

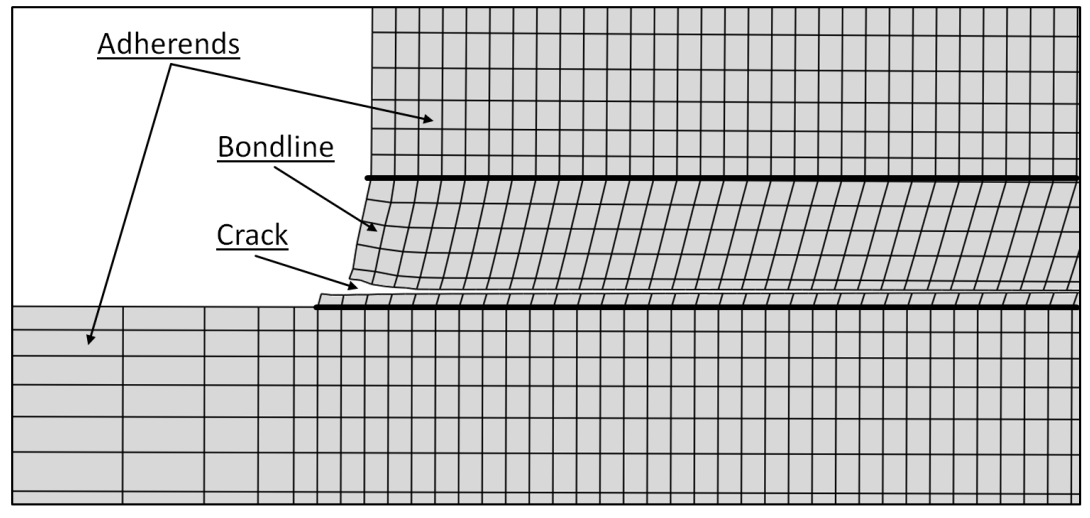

Figure 12: Crack in XFEM-analysis with exp. Drucker-Prager model and $t_{b}=0.125 \mathrm{~mm}$

from the numerical simulations are shown. Supplementary, the range of the experimental failure loads is incorporated in the figure. The different simulation configurations with the same material model differ only in terms of the bondline disrectisation from each other. The load-displacement data of all simulations are nearly congruent until failure. Although the failure loads differ from each other, independent of the discretisation the failure points of each material model lie closely together. Depending on the bondline thickness, simulations with the exponent and the von Mises yield criterion are closest to the experimental data. 


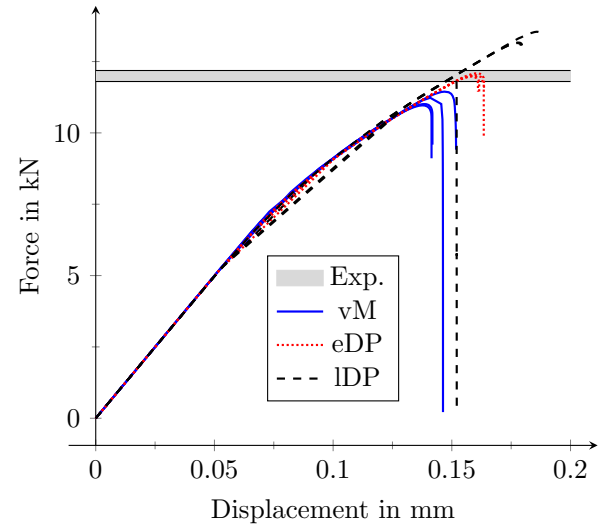

(a) $t_{b}=0.125 \mathrm{~mm}$

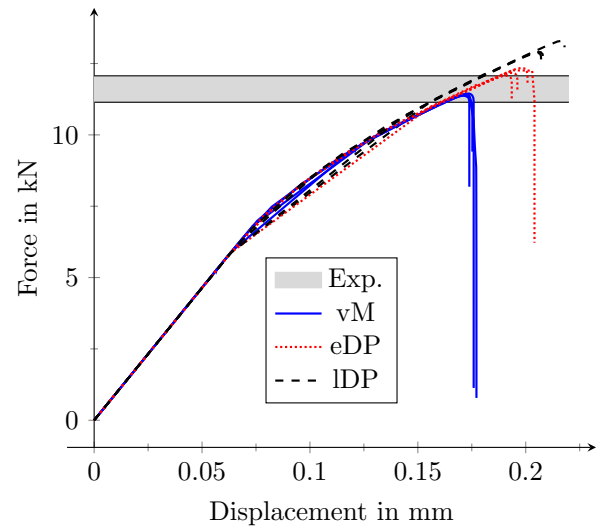

(b) $t_{b}=0.21 \mathrm{~mm}$

Figure 13: Force-Displacement data from numerical simulations

the determined strengths have always the greatest difference to the experiments and are on top of that non-conservative.

Because there is no setting from the clamping fixture and no stiffness of the testing machine in the simulations, the load-displacement data cannot be campared directly to the data given in section 4.1. To be able to evaluate the numerical strength prediction quantitatively, the difference between experimental and numerical failure load has been calculated with equation 4.1 .

$$
\text { Difference } \%=\frac{\mid \text { Experimental }- \text { Numerical } \mid}{\text { Experimental }} \cdot 100
$$

The results for the different configurations are summarised in table 5 . Some simulations did not converge with the standard settings. To achieve convergence, in simulations marked with * the damage initiation tolerance was increased from 5 $\%$ to $7.5 \%$. If the calculated stress in an increment is greater than the specified damage initiation stress, the increment size will be reduced and the increment has to be recalculated. The tolerance specifies how much the specified initiation stress can be exceeded without recalculating the increment. If this measure was not sufficient to achieve convergence, a viscous regularization with a coefficient of $1 \cdot 10^{-5}$ was additionally applied. These simulations are marked with **. Both measures could result in a lower accuracy of the solutions. 
The numerical predicted strengths of the TAST specimen with the thin bondline deviate between $0.01 \%$ and $12.93 \%$ from the experimental results. In contrast to an underestimated strength from the von Mises criterion the linear and the exponent Drucker-Prager overestimate the strength. While the von Mises models give conservative strength predictions with a variance to the experimental findings between $4.57 \%$ and $8.58 \%$, the strengths predicted with the exponent Drucker-Prager model are with a variance to the experiments of less than 1

${ }_{345} \%$ fairly accurate. The linear Drucker-Prager model gives the widest range of strength values and has the greatest difference to the experiments. The outlier with one element in through-thickness direction could be attributed to the artificial damping.

By and large, the simulations for the thick bondline show the same findings 350 except that the von Mises' and not the exponent Drucker-Prager yield criterion gives the most accurate predictions. Nevertheless, the strength predictions with the exponent Drucker-Prager criterion are with differences between $4.02 \%$ to $6.31 \%$ still very precise.

Moreover, it can be stated that there is no recognisable correlation between a finer mesh and a more accurate strength prediction. As a starting point, five elements through-thickness is a good value since all of the simulations with this discretisation showed good convergence behaviour and a smaller computation time than the seven element through-thickness models. 
Table 5: Comparison of experimental and numerical failure loads

\begin{tabular}{cc|cc|cc} 
& & $t=0.125 \mathrm{~mm}$ & & $t=0.21 \mathrm{~mm}$ & \\
\hline & Elem. & $\begin{array}{c}\text { Failure Load } \\
\text { in N }\end{array}$ & $\begin{array}{c}\text { Diff. } \\
\text { in \% }\end{array}$ & $\begin{array}{c}\text { Failure Load } \\
\text { in N }\end{array}$ & $\begin{array}{c}\text { Diff. } \\
\text { in \% }\end{array}$ \\
\hline Exp. & - & $11993 \pm 193$ & - & $11607 \pm 463$ & - \\
\hline vM & 1 & $11231^{* *}$ & 6.36 & $11412^{* *}$ & 1.68 \\
vM & 3 & $11445^{* *}$ & 4.57 & 11386 & 1.91 \\
vM & 5 & 10964 & 8.58 & 11449 & 1.36 \\
vM & 7 & 11010 & 8.20 & 11377 & 1.98 \\
\hline lin. DP & 1 & $12000^{* *}$ & 0.06 & 12129 & 4.50 \\
lin. DP & 3 & $13544^{*}$ & 12.93 & $13303^{*}$ & 14.62 \\
lin. DP & 5 & 13165 & 9.77 & 12909 & 11.21 \\
lin. DP & 7 & 13149 & 9.64 & 12871 & 10.89 \\
\hline exp. DP & 1 & 12038 & 0.38 & $12262^{*}$ & 5.64 \\
exp. DP & 3 & 12087 & 0.78 & 12343 & 6.31 \\
exp. DP & 5 & 11992 & 0.01 & 12171 & 4.86 \\
exp. DP & 7 & 11977 & 0.13 & 12073 & 4.02
\end{tabular}

\section{Conclusion}

The capability of XFEM for the strength prediction of lap joints bonded with a thin film adhesive was investigated in this work. All material parameters used, were derived from specimen with one adhesive film layer and a bondline thickness of about $t_{b}=0.1 \mathrm{~mm}$. With this material data and $2 \mathrm{D}$ simulation models strength predictions of TAST specimen with bondline thicknesses $t_{b}=$ $0.1 \mathrm{~mm}$ and $t_{b}=0.2$ were carried out.

The following conclusions can be drawn:

1. XFEM in combination with a suitable yield criterion is a valid technique for the prediction of lap shear failure loads since the variance to the experiments is in the present case always less than $10 \%$.

2. Prediction of the joint failure load under shear loading with the exponent Drucker-Prager model is very accurate, especially when using material data which are derived from specimen with the same bondline thickness like the joint to be predicted.

3. There is no clear indication regarding the discretisation. However, a discretisation of three to five elements in through-thickness direction of the 
adhesive bondline can be recommended as a starting point for XFEM strength predictions of mainly shear loaded joints.

4. If not all experimental values needed for the exponent Drucker-Prager model are available, the von Mises yield criterion is a suitable alternative since the predicted values for the TAST specimen are always conservative.

5. The linear Drucker-Prager model is not well suitable in the present case, which underlines the findings of Dean et al. [30].

It could be shown that XFEM in combination with a well chosen material model for the adhesive is a suitable tool for strength predictions of shear loaded bonded joints. This method can be used for strength predictions in global as well as in detail design of bonded structures. Notwithstanding, the slight difference between the strains from DIC and FEA must be investigated further. Moreover, the dependence of the prediction accuracy on the bondline thickness needs further research.

\section{Acknowledgements}

The authors would like to thank the European Commission by funding this work through the Clean Sky 2 research programme.

\section{References}

[1] Dirschmid, F. Die CFK-Karosserie des BMW i8 und deren Auslegung. In Karosseriebautage Hamburg; Springer: Hamburg, Germany, 2014.

[2] Zarouchas, D.; Nijssen, R. J. Adhes. Sci. Technol. 2016, 30, 1413-1429.

[3] Gramüller, B.; Stroscher, F.; Schmidt, J.; Ungwattanapanit, T.; Löbel, T.; Hanke, M. Design process and manufacturing of an unmanned blended wing-body aircraft. In Deutscher Luft-und Raumfahrtkongress; 
[4] da Silva, L. F.; das Neves, P. J.; Adams, R.; Spelt, J. Int. J. Adhes. Adhes. 2009, 29, 319-330.

[5] da Silva, L. F.; das Neves, P. J.; Adams, R.; Wang, A.; Spelt, J. Int. J. Adhes. Adhes. 2009, 29, 331-341.

[6] Rodríguez, R. Q.; de Paiva, W. P.; Sollero, P.; Rodrigues, M. R. B.; de Albuquerque, É. L. Int. J. Adhes. Adhes. 2012, 37, 26-36.

[7] He, X. Int. J. Adhes. Adhes. 2011, 31, 248-264.

[8] Jia, Z. M.; Yuan, G. Q.; Hui, D. Adv. Mater. Res. 2014, 1049, 892-900.

[9] Özer, H.; Öz, Ö. Int. J. Adhes. Adhes. 2017, 76, 17-29.

[10] Tomblin, J.; Strole, K.; Dodosh, G.; Ilcewicz, L. "Assessment of industry practices for aircraft bonded joints and structures", Technical Report DOT/FAA/AR-05/13, Federal Aviation Administration, Washington, 2005.

[11] Pascoe, J.; Alderliesten, R.; Benedictus, R. Eng. Fract. Mech. 2013, 112, $72-96$.

[12] de Sousa, C.; Campilho, R.; Marques, E.; Costa, M.; da Silva, L. Proc. Inst. Mech. Eng. Part L: J. Mater. Des. 2017, 231, 210-223.

[13] Blackman, B. Int. J. Fract. 2003, 119, 25-46.

[14] Krueger, R. Appl. Mech. Rev. 2004, 57, 109-143.

${ }_{420}$ [15] Mubashar, A.; Ashcroft, I. J. Adhes. 2017, 93, 444-460.

[16] Campilho, R. D.; Banea, M. D.; Pinto, A. M.; da Silva, L. F.; De Jesus, A. Int. J. Adhes. Adhes. 2011, 31, 363-372.

[17] Mubashar, A.; Ashcroft, I.; Crocombe, A. J. Adhes. 2014, 90, 682-697.

[18] Stuparu, F.; Constantinescu, D.; Apostol, D.; Sandu, M. J. Adhes. 2016, 425 $92,535-552$. 
[19] Xará, J.; Campilho, R. Compos. Struct. 2018, 183, 397-406.

[20] Mises, R. v. Nachrichten von der Gesellschaft der Wissenschaften zu Göttingen, Mathematisch-Physikalische Klasse 1913, 1913, 582-592.

[21] Drucker, D. C.; Prager, W. Q. Appl. Math. 1952, 10, 157-165.

[22] ASM Handbook Committee, Metals Handbook: Properties and Selection: Nonferrous Alloys and Special-Purpose Materials; volume 2 of ASM Handbook ASM International: Materials Park, OH, USA, 10 ed.; 1990.

[23] Holzhüter, D.; Wilken, A. "Einfluss von Umgebungsbedingungen auf die Festigkeit geklebter lagenvariabler Schäftverbindungen", Interner Bericht IB 131-2015 / 52, DLR-Institut für Faserverbundleichtbau und Adaptronik, Braunschweig, 2015.

[24] Kosmann, J.; Klapp, O.; Holzhüter, D.; Schollerer, M.; Fiedler, A.; Nagel, C.; Hühne, C. Int. J. Adhes. Adhes. 2018, 83, 50-58.

[25] Nagel, C.; Klapp, O. Yield and Fracture of Bonded Joints using HotCuring Epoxy Film Adhesive - Multiaxial Tests and Theoretical Analysis. In International Symposium on Sustainable Aviation; ISSACI: Rome, Italy, 2018.

[26] Floros, I.; Tserpes, K.; Löbel, T. Composites Part B 2015, 78, 459-468.

[27] "ASTM D5656-10: Standard test method for thick-adherend metal lapshear joints for determination of the stress-strain behavior of adhesives in shear by tension loading", 2017.

[28] Henkel Corporation Aerospace, "LOCTITE EA 9695 AERO Epoxy Film Adhesive", Technical Process Bulletin, 2013.

[29] Kosmann, J.; Völkerink, O.; Schollerer, M. J.; Holzhüter, D.; Hühne, C. "Digital image correlation strain measurement of thick adherend shear test specimen joined with an epoxy film adhesive", 2018 submitted to Int. J. Adhes. Adhes. 
[30] Dean, G.; Read, B. E.; Duncan, B. "An evaluation of yield criteria for adhesives for finite element analysis", NPL Report CMMT(A)117, National Physical Laboratory, Teddington, United Kingdom, 1999.

[31] Schollerer, M. J.; Kosmann, J.; Löbel, T.; Holzhüter, D.; Hühne, C. Appl. Adhes. Sci. 2017, 5-15.

[32] Dean, G.; Crocker, L. "The use of finite element methods for design with adhesives", Measurement Good Practice Guide No. 48, National Physical Laboratory, Teddington, United Kingdom, 2001.

[33] Wang, C. H.; Chalkley, P. Int. J. Adhes. Adhes. 2000, 20, 155-164.

[34] Belytschko, T.; Black, T. Int. J. Numer. Meth. Eng. 1999, 45, 601-620.

[35] Belytschko, T.; Moës, N.; Usui, S.; Parimi, C. Int. J. Numer. Meth. Eng. 2001, 50, 993-1013.

465 [36] Dassault Systemes Simulia Corp., "Abaqus 2016", Johnston, RI, USA,.

[37] da Silva, L. F.; Lopes, M. J. C. Int. J. Adhes. Adhes. 2009, 29, 509-514.

[38] Öz, Ö.; Özer, H. J. Adhes. Sci. Technol. 2017, 31, 2251-2270.

[39] Da Silva, L. F.; Rodrigues, T.; Figueiredo, M.; De Moura, M.; Chousal, J. J. Adhes. 2006, 82, 1091-1115.

${ }_{470}$ [40] Jouan, A.; Constantinescu, A. Int. J. Adhes. Adhes. 2018, 84, 63-79. 